\title{
Consideraciones de las dinámicas y procesos de cambio de población y del ambiente para el desarrollo territorial con un sistema alimentario y nutricional sostenible Centroamericano
}

\section{Considerations of the dynamics and processes of population change and the environment, for territorial development with a Central American sustainable food and nutrition system}

\author{
Adolfo Martín Hurtado Díaz ${ }^{1,}$ Mauricio Jesús Córdoba Salinas ${ }^{2}$ \\ ${ }^{1}$ ORCID: https://orcid.org/0000-0003-0238-6405, adolfohurtado@consultant.com \\ 2 ORCID: https://orcid.org/0000-0001-9127-0193,mcordobasalinas@gmail.com \\ Investigadores independientes
}

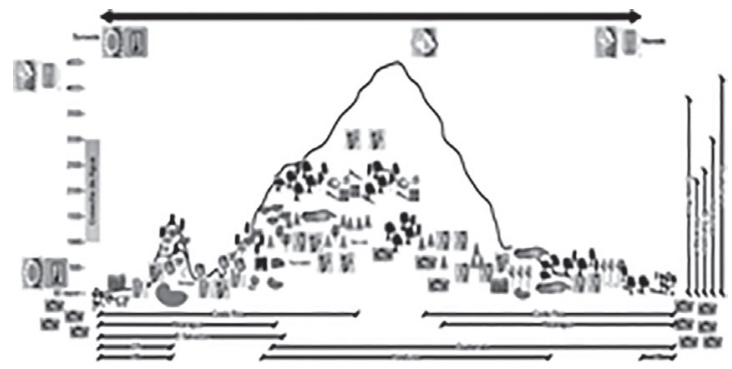

\section{RESUMEN}

Los análisis de dinámicas y procesos de cambio territorial nos permiten interpretar y dar sentido a la articulación entre los diferentes elementos constitutivos de los ámbitos del desarrollo y cómo estos se han generado, integrando en los procesos una visión dinámica y prospectiva muy pertinente para abordajes integrales, incluyentes y sostenibles del desarrollo territorial. En el presente escrito se abordan los ámbitos de población y del ambiente desde una perspectiva regional.

Palabras clave: consumo, nutrición, clima, biodiversidad, políticas.

\section{ABSTRATC}

The analysis of dynamics and processes of territorial change allow us to interpret and understand the articulation between the different elements of the development spheres and how they have been generated, integrating into the process a dynamic and prospective vision that addresses the areas of population and the environment from a regional perspective.

Keywords: consumption, nutrition, climate, biodiversity, policies.

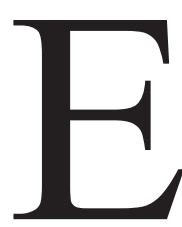
1 sistema alimentario centroamericano y el desarrollo rural que lo sustenta enfrentan condiciones adversas para su sostenibilidad a diferentes niveles territoriales. En el nivel nacional nos enfocamos en las condiciones generales de este sistema alimentario regional y del modelo de desarrollo rural de los últimos 60 años, que lo tensionan y amenazan. En este escrito se abordan procesos y dinámicas de población y del ambiente desde una visión regional.

Consideraciones de la población. Según Informe técnico de tendencias de la disponibilidad y la necesidad de alimentos en los países de la región del PRESISAN (Sibrián y Palma, 2013), para 2050 se proyecta un incremento de población que oscila entre el $60 \%$ (con baja fecundidad) a por debajo del $90 \%$ (con alta fecundidad). Esto demanda el desarrollo de estrategias de incremento de disponibilidad alimentaria para el abastecimiento Per cápita, por volumen y diversidad, así como por mejoría en los sistemas de comercio, abastecimiento y consumo.

En las últimas décadas, las ciudades pequeñas y de medio porte han sido receptoras de la migración rural (Berdegué y Meynard, 2012). A falta de un desarrollo adecuado estas han sido sensibles a los fenómenos de migración y violencia, y hay cambios en la estructura poblacional local, Por ejemplo, el Ministerio de Relaciones
Recibido: 2 de abril del 2019

Aceptado: 26 de junio del 2019

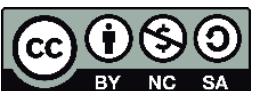

Los artículos de la revista La Calera de la Universidad Nacional Agraria, Nicaragua, se comparten bajo términos de la licencia Creative Commons: Reconocimiento, No Comercial, Compartir Igual. Las autorizaciones adicionales a las aquí delimitadas se pueden obtener en el correo edgardo.jimenez@ci.una.edu.ni

C Copyright 2019. Universidad Nacional Agraria 
Exteriores de El Salvador tiene una tipología de ciudades según su estructura poblacional, y en función de eso, establece las estrategias de desarrollo que apoyan; esto hace pensar que las ciudades pequeñas y de mediano porte serán esenciales en el desarrollo sostenible de Centroamérica.

Centroamérica en general, y cada uno de sus países con sus particularidades, enfrentan cuatro cambios poblacionales sustanciales, que está modificando las condiciones de desarrollo general y de nuestro sistema alimentario y ruralidad en particular. Estos cambios están vinculados al crecimiento de la población urbana, la migración y el envejecimiento.

Nicaragua hizo su transición de estructura de población de rural a urbano en la segunda mitad de la década de los 70, seguido por Costa Rica y El Salvador en los 80, la región centroamericana la vivió en los 90, y Honduras y Guatemala en la segunda mitad de la década pasada. Esta transición fue acompañada por profundos cambios en la cultura alimentaria reduciendo su diversidad y los conocimientos de gestión de su estacionalidad, así como en las condiciones de salud de la población con incremento de la obesidad (Hurtado, 2015).

En este cambio poblacional se ha presentado una creciente capacidad de selección; Dumazert (2008) expresa que la tendencia creciente de mejoría de ingreso general de la población va acompañada de un incremento de opciones para elegir, dentro de un perfil de productos o nuevos perfiles de consumo; y la calidad de su cultura alimentaria representada por las preferencias, y conocimientos en sus decisiones al escoger (capacidad de elección). Estos ámbitos tienen un papel creciente en la promoción de la Soberanía, Seguridad Alimentaria y nutricional de la región y sus países. Por ello el desarrollo de políticas que fortalezcan la cultura alimentaria para la gestión de la biodiversidad y su estacionalidad, es de profunda importancia para la protección de la producción agroalimentaria y el consumo regional. Así también junto al proceso de urbanización se hace necesaria, cada vez más, la promoción de estilos de vida saludables.

Otro factor que tuvo impacto, en la organización poblacional, fue la implementación de la revolución verde en Centroamérica: resultó en un cambio societal; Hurtado (2005) hace referencia a cambios en las estructuras generales nacionales (urbano/rural), profunda diversificación de la tipología de actores y jerarquías sociales en lo urbano y rural, monetización de la economía rural, cambios en los instrumentos y modelos de gobernanza con la construcción de los Estados nacionales; de fuertes movimientos de población en diferentes niveles territoriales (urbano-rural, de valles a laderas, de territorios económicamente integra- dos a las fronteras agrícolas; y entre países como el mercado regional de cortadores de algodón, en un inicio) en los últimos 60 años (Hurtado, 2015). Estos cambios fueron acompañados por la construcción de nuevas identidades, entre ellas las reivindicativas de mujeres por la igualdad de derechos. Un resultado ha sido una mayor feminización de lo rural que se expresa hoy en un mayor número relativo de mujeres al frente de unidades productivas y de servicios, así como en la fuerza laboral rural (Lastarria-Cornhiel, 2008). Sin embargo, la equidad en el acceso de recursos, servicios e información es una tarea pendiente. Estrategias de inclusión con equidad de género tiene una importancia estratégica creciente para un sistema alimentario y desarrollo territorial sostenible, además de materia pendiente en derechos económicos, sociales y culturales.

Los países de Centroamérica se encuentran en diversos estados de avances en su bono demográfico (Estado de la Región, 2016). Costa Rica y Panamá se encuentran en la fase de agotamiento de este proceso que se proyecta para el 2020. Se proyecta que El Salvador llegará al final de este período hacia el 2030. Belice, Nicaragua y Honduras tiene hasta el 2035 y Guatemala (el único país que se encuentra al inicio) se proyecta hacia el 2050. Para la obtención de resultados favorables "es necesario realizar esfuerzos en materia educativa y laboral, con el fin de que las personas en edad de trabajar se conviertan en económicamente activas y altamente productivas" (Estado de la Región, 2016, p. 106). Una vez terminado el bono demográfico, el envejecimiento de la población vendrá acompañado de una reducción relativa de población económicamente activa que de no ser compensada con adecuados incrementos de productividad o con ingresos no provenientes del trabajo como el ahorro, podría provocar efectos negativos en el bienestar económico. En este contexto el incremento de los salarios mínimos por encima de la canasta básica, capaces de garantizar la seguridad social para toda la población, es una meta estratégica, que dispone de relativamente poco tiempo, así como la protección universal del adulto mayor.

Así también, encontramos que la edad promedio de los productores de granos básicos de Centroamérica a mitad de la década pasada era de 49 años y 45 años de la población rural (Baumeister, 2010). Para esto se hace necesario el desarrollo de una nueva economía territorial incluyente, que resulte atractivo a jóvenes.

Centroamérica, como Mesoamérica y la región del SICA, está construida sobre una gran base multicultural, de origen (originarios, europeos, africanos y orientales) y fusiones (creole, garífunas, mestizos, mulatos, ...), ocupando toda la diversidad territorial de la región (Atlas de Centroamérica, 2011). Son culturas que llevan siglos ocupando sus 
actuales territorios y que han acumulado conocimiento a lo largo de todo ese tiempo, cada una de ellas con sus visiones y valores. Esto demanda que toda construcción de desarrollo inclusivo y sostenible parte de diálogo de saberes que se conformen comunidades epistémicas.

Consideraciones ambientales. Centroamérica presenta una alta diversidad biológica y nichos ambientales en una extensión geográfica relativamente reducida. Conformada por diferencias importantes en altura (desde el nivel del mar hasta entorno de $4500 \mathrm{msnm}$ ), de zonas climáticas (Cuenca del Pacífico seca - hasta mínimos de $800 \mathrm{~mm}$ año${ }^{1}$ y cuenca del Caribe húmeda - hasta máximos de 5000 $\mathrm{mm}$ año ${ }^{-1}$ ), con gran variedad de suelos en su constitución y antigüedad (de calcáreos a volcánicos) que se expresan en regiones o zonas ecológicas diversas (Atlas Centroamericano, 2011). Pero, además, Centroamérica geológicamente fue puente entre la flora y fauna de América del Norte (mamíferos y plantas con flores) y América del Sur (marsupiales y plantas sin flores) hace unos 33.5 millones de años, lo que le da una gran diversidad biológica por unidad de área en relación con otras áreas del planeta. Todo esto conforma el Hotspot -área o territorio donde hay una especial concentrción de biodiversidad- de Mesoamérica, lo que implica que con "menos del 0.5 por ciento de la extensión terrestre del planeta, Mesoamérica posee entre el 7 y el 10 por ciento de todas las formas de vida conocidas y el 17 por ciento de todas las especies terrestres" (Critical Ecosystem Partnership Fund, 2004). Cualquier modelo de desarrollo que pretenda ser sostenible y que quiera aprovechar el potencial territorial de desarrollo, debe de partir de la gestión social de esta diversidad biológica y ambientales. Esta condición se presenta como una oportunidad para el incremento de la disponibilidad de alimentos por diversidad de la biota alimentaria disponible, frente a la perspectiva de crecimiento de población. Esta condición genera la necesidad de tener la capacidad de gestionar adecuadamente la biodiversidad y su estacionalidad, donde una adecuada cultura alimentaria y sistemas inteligentes de información son centrales en esta gestión.

Los países del SICA se construyen sobre territorios con pendientes que conforman hasta el $70 \%$ de su superficie (Atlas de Centroamérica, 2011), lo que implica espacios productivos ambientalmente frágiles en sus ciclos de agua y suelo, con significativas variaciones de disponibilidad de agua (inter e intra anual) y temperaturas según altura. Esta condición orienta a necesidades de desarrollo tecnológico-económico-ambiental para la producción agroalimentaria y no alimentaria a modelos productivos eficientes en la gestión del agua y la humedad, basados en coberturas forestales con una pecuaria adecuada a las condiciones ambientales.

En relación con la superficie agricultable de la región (FAO STAT, datos del 2009), sólo Nicaragua cuenta con una superficie agricultable por habitante $(0.33 \mathrm{ha} /$ hab) mayor a la media mundial ( 0.2 ha/hab), Centroamérica cuenta con una disponibilidad menor $(0.14 \mathrm{ha} / \mathrm{hab}) \mathrm{a}$ la media mundial, y como SICA, esta área disponible se reduce $(0.13 \mathrm{ha} / \mathrm{hab})$, esto tiene como implicación fuertes restricciones de superficie disponible por trabajador(a). Esto demanda estrategias intensivas en resultados por unidad de superficie (producción de biomasa útil - alimentaria y no alimentaria) e intensiva en recurso humano (trabajo y conocimiento) para una optimización social del modelo de desarrollo.

Existe una una dotación diferenciada de recursos entre los países Centroamérica como por ejemplo: Nicaragua es el país que cuenta con más superficie agricultable por habitante y Costa Rica menos (1/8 respecto a Nicaragua); Costa Rica y Guatemala con mayor dotación de biodiversidad productiva alimentaria y Nicaragua con el menor ( $2 / 5$ de la biodiversidad productiva); todo el territorio salvadoreño se encuentra en el corredor seco y sus principales cosechas de agua se encuentran fuera de su territorio. Lo anterior hace estratégico fortalecer el comercio agroalimentario regional como comercio comunitario, promover la sostenibilidad de las aguas de la región y fortalecer su cultura alimentaria para gestionar su biodiversidad alimentaria y con eficiencia su estacionalidad.

Cuadro 1. Dotación de recursos para la producción agroalimentaria

\begin{tabular}{lcccccc}
\hline & GT & HN & SV & NI & CR & CA \\
\hline Total de variedades de productos & 42 & 38 & 29 & 18 & 43 & 59 \\
$\begin{array}{l}\text { Producto en relación al total regional } \\
\text { de variedades de productos (\%) }\end{array}$ & 71 & 64 & 49 & 31 & 73 & -- \\
Productos únicos & 7 & & 2 & & 8 & -- \\
Dos países con ese producto & 6 & 7 & 3 & & 6 & -- \\
\hline
\end{tabular}

Gt: Guatemala, HN: Honduras, SV: El Salvador, NI: Nicaragua, CR: Costa Rica, CA: Centro América.

Fuente: Hurtado, (2015).

El cambio climático. Diversos autores expresan que el cambio climático se experimentará en Centroamérica con una disminución en precipitación anual, una menor cantidad de días con lluvia, aparejado de incremento de temperatura media, así como exacerbaciones en la variabilidad climática. Esto podrá llevar en diversos territorios de Centroamérica a reducción de disponibilidad de agua y reducción de biomasa, principalmente de aquellas cuen- 
cas hidrográficas que alcanzan alturas superiores a los 800 msnm. Lo anterior hace necesario la reducción de riesgos incrementando la gestión de la biodiversidad alimentaria y su estacionalidad (productiva y cultural), y estrategias territoriales para la gestión integral y eficiente del agua, que proteja la producción y el consumo.

El grado de afectación de la carencia de agua también depende de las alturas de las microcuencas. Las que alcancen alturas mayores a los $1000 \mathrm{msnm}$, podrán beneficiarse del efecto adiabático de las corrientes de aire. Entre los 800 y $1000 \mathrm{msnm}$ podrán beneficiarse de la neblina en los días más fríos. Las comunidades sin microcuencas, que superen los $800 \mathrm{msnm}$, serán las más afectadas. Una estrategia de construcción de economía ambiental basada en la cobertura forestal y la biodiversidad puede desarrollarse a lo largo de todo el año, inclusive en períodos del fenómeno de El Niño, en alturas superiores a las 1000 a $1100 \mathrm{msnm}$. Por debajo de los $1000 \mathrm{msnm}$ tendrán que alternarse años secos para trabajo (Los programas de Alimentos por Trabajo o sus equivalentes, pueden orientarse al desarrollo de esta infraestructura hidráulica regional) en micro cuencas y cuencas (para cosecha y estabilización de aguas) y los períodos húmedos para incrementar la biomasa boscosa. Mejoría de condiciones en micro cuencas arriba de los $1000 \mathrm{msnm}$ podrían redundar en mejoría en la cantidad de tiempo disponible para la recomposición de la economía ambiental entre los 800 y $1000 \mathrm{msnm}$. De los resultados de la economía ambiental en las laderas, dependerán en gran medida los resultados de la economía ambiental y la seguridad de las costas (salud de sus man- glares), las planicies y los valles (manto acuífero y calidad de los suelos). Esto hace necesario una política de Estado de los países de Centroamérica de promoción y desarrollo de una economía de cobertura forestal, así como de gestión de biodiversidad y agua. Será necesaria una economía ambiental basada en la cobertura forestal (sistemas agroforestales, silvopastoriles o de forestaría comunitaria) para adaptarse a estos cambios, protegerse y convertirlos en una oportunidad. Para el desarrollo de la agroindustria, debe de considerarse la eficiencia en la gestión del agua.

Una de las características ya observadas en el cambio climático es la intensidad y duración de los eventos extremos, como observados con los Fenómenos de El niño y la Niña. Con estos escenarios y de mantenerse el actual modelo de desarrollo rural, habrá conflictos entre los distintos consumos de agua (humano, animal y riego). Esto demanda que el desarrollo territorial y el sistema alimentario necesitan estar y desarrollarse territorialmente adecuado.

\section{AGRADECIMIENTOS}

Estas consideraciones se han realizado en el marco del proceso de investigación de proyectos relacionados con el desarrollo de la plataforma PECOSOL - CONSUACCIÓN para la Seguridad Alimentaria y Nutricional en Centroamérica. Los autores agradecen a OIKOS - Cooperação e Desenvolvimento por su compromiso en la región centroamericana y muy especialmente a la Fundación Calouste Gulbenkian y Camões - Instituto de la Cooperación y la Lengua IP por su apoyo financiero para la realización de los estudios.

\section{REFERENCIAS BIBLIOGRÁFICAS}

Baumeister, E. (2010). Pequeños productores de granos básicos en América Central. Cuantificación, caracterización, nivel de ingresos, pobreza, y perfiles demográficos, socioeconómicos y ocupacionales. FAO-RUTA Honduras.

Berdegué, J., y Meynard, F. (2012). Las Ciudades en el Desarrollo Territorial Rural. Serie Claves para el Desarrollo Territorial. Santiago Chile: RIMISP-Centro Latinoamericano para el Desarrollo Rural.

Critical Ecosystem Partnership Fund. (2004) Perfil de Ecosistema. Región sur del Hotspot de biodiversidad en Mesoamérica (Belice, Guatemala y México). Recuperado de https://www.cepf.net/sites/default/files/final.spanish.mesoamerica.northernmesoamerica.ep_. pdf

Estado de la Región. (2016). Quinto Informe. Costa Rica. Recuperado de http://www.estadonacion.or.cr/erca2016/assets/erca-2016web.pdf

Hurtado, A. (2015). Estado de la Soberanía Alimentaria de Centroamérica: Repensando la gobernabilidad regional para un desarrollo rural incluyente, integrado y sostenible frente a los grandes retos de la globalización. ADHAC.

Lastarria-Cornhiel, S. (2008). Feminización de la agricultura en América Latina y África Tendencias y fuerzas impulsoras. Serie DEBATES Y TEMAS RURALES N ${ }^{\circ} 11$. Santiago Chile: RIMISP-Centro Latinoamericano para el Desarrollo Rural.

Sibrián, R., y Palma, P. (2013). Tendencias de la disponibilidad y la necesidad de alimentos en los países de la región del SICA. Informe técnico, OBSAN-R PRESISAN. Recuperado de file://D:/Datos\%20de\%20Usuario/Descargas/Tendencias\%20de\%20la\%20disponibilidad $\% 20 \mathrm{y} \% 201 \mathrm{la} \% 20$ necesidad $\% 20 \mathrm{de} \% 20$ alimentos $\% 20$ en $\% 201$ os $\% 20$ paises $\% 20 \mathrm{de} \% 201 \mathrm{a} \% 20$ region $\% 20 \mathrm{del} \% 20$ SICA $\% 20$ \%20Mayo\%202013.pdf

Sistemas de Información FAO; FAOSTAT. Dirección de Estadística. Recuperado de http://faostat3.fao.org/home/S 